\title{
Washing of Fabrics
}

\author{
Part 4: Experiments and Analysis on Washing Polyester Cotton Blended Fabrics to \\ Remove Polyvinyl Alcohol
}

By Hitoshi Matsui, Shigenori Fukuoka, Shigeo Yoshida, Members, TMSJ

Based on the Journal of the Textile Machinery Society of Japan, Transactions, Vol. 31, No. 7, T109-T118 (1978)

\begin{abstract}
The effects of concentration of polyvinylalcohol (PVA) in washing bath, washing number, time, temperature, on washing quality in repeated washing were experimentally investigated with polyester/ cotton blended fabrics treated with PVA. These experimental results were compared with calculated values using the theoretical equations ${ }^{[1,2]}$, previously derived based on the diffusion theory and a newly derived theoretical equation which referred to the effect of the bath concentration of a chemical to be removed. Assuming that PVA does not exist in fibers but only among fibers and that the fiber assembly is a column, the diameter of which is the same as that of the yarn, it has been found that the above mentioned experimental results agree well with the theoretical ones.

Further, according to these results the washing processes in a practical washing machine were analyzed in detal. Under the same assumptions, it has also been found that the calculated values based on this theoretical analysis agree well with experimental results obtained by using a practical washing machine. According to these studies, the approximate washing efficiencies using a practical washing machine, can be predicted from the fundamental values such as the diffusion coefficient of a chemical to be removed, the yarn diamer, etc.
\end{abstract}

\section{Introduction}

In spite of the importance of the washing process in dyeing and finishing, a limited number of studies have been hitherto conducted. In Japan, only the works by Konishi and Waki$\mathrm{ta}^{[5-13]}$ are notable.

We have investigated the diffusion of chemicals in fibers or in water filling interstices of the fiber assembly and set up theoretical equations in washing ${ }^{[1,2]}$. In this paper we carried out washing experiments using polyester/cotton blended fabrics treated with polyvinyl alcohol (PAV) and compared the results with the theoretical equations above mentioned.

The washing process in dye houses is more or less characterized by repeated soaking-squeezing processes, and the liquor is contaminated by substances washed out from textiles. Studies on the effect of repeated washing and contaminated wash liquor on the washing efficiency are very scarce except the theoretical treatments by Parish ${ }^{[15]}$, Bonkáls ${ }^{[16]}$, Ratna Prabhu ${ }^{[17]}$ and Matsui et al. ${ }^{[3,4]}$.

In a continuous washing machine, the concentration of a chemical in the wash bath at the beginning of washing is approximately zero. As the process goes on, this concentration increases, and finally reaches a constant and the chemical from the fiber assembly balances with the chemical discharged from the wash bath. We call this the "dynamic "equilibrium". Parish ${ }^{[15]}$ and Bonkáls ${ }^{[16]}$ reported the dynamic equilibrium in washing, but paid little consideration of the squeezing effect. The equations derived by them can be applied only to such a squeezing ratio as is equal before and after the soaking, and only to fabrics with similar water contents. They expounded the preferential absorption of chemicals on fibers which rather fits under a special case and is not a general one.

Prabhu ${ }^{[17]}$ studied washing efficiency by investigating the effect of squeezing and soaking separately. However, he did not treat both the preferential absorption of chemicals onto the fiber assembly and the concentration change of chemicals in the washing bath.

In the previous papers ${ }^{[3,4]}$ we treated such experiments as repeated washing of cotton fabrics added with caustic, soda, or as washing in caustic soda solutions of various concentration. Then, we analyzed these data and discussed the washing in a multi-both washing machine the dynamic equilibrium based on the uptake and the discharge rate of chemicals under the assumption that the water contents in the fabric before washing and after squeezed are equal. In addition, we did not elaborate the preferential absorption of chemicals onto the fiber.

In this paper we carried out such experiments as repeated washing of polyester/cotton blended fabrics added with PVA or as washing experiments in PVA solutions of various concentration. We analysed these data taking into considerration such factors as the preferential absorption of chemicals onto fibers and the squeezing before and after washing. Subsequently, we analysed the washing performance in a 
multi-bath washing machine by the results obtained above and from the point of material balance. We treated not only a dynamic equilibrium state but also the conditions under which washing bath concentration is changing. Finally we compared the results with those obtained from a four-bath washing machine in a dye house.

\section{Experimental Methods}

\subsection{Samples}

The characteristics of fabrics used in this experiment are shown in Table 1. The polyester/cotton blended fabrics were

Table 1 Fabrics used

\begin{tabular}{lc}
\hline Structure & Plane \\
Yarn count Warp (NE) & 45 \\
Yarn count Filling (NE) & 45 \\
Density Warp (ends/in) & 110 \\
$\quad$ Filling (picks/in) & 76 \\
Unfinished fabrics Width $(\mathrm{cm})$ & 115.5 \\
Unfinished fabrics Weight $(\mathrm{g} / \mathrm{m})$ & 116 \\
\hline
\end{tabular}

desized by enzyme, scoured by soda ash and then bleached by hydrogen peroxide. For the washing experiments in the practical washing machine, unfinished fabrics of the same characteristics were utilized. These fabrics contained $4.8 \%$ PVA 217 obtained from Kuraray Co. with a degree of polymerization $=1700$.

\subsection{Basic Washing Experiment}

The above fabric samples were cut into one-gram pieces and soaked in $20^{\circ} \mathrm{C}, 4.0 \%$ PVA 217 solution for 60 seconds. After sufficient permeation, the fabrics were squeezed by a mangle, dried at $90^{\circ} \mathrm{C}$ for 7 minutes and then washed. The squeezing ratio of the mangle used was $88.2 \%$ to the abso-

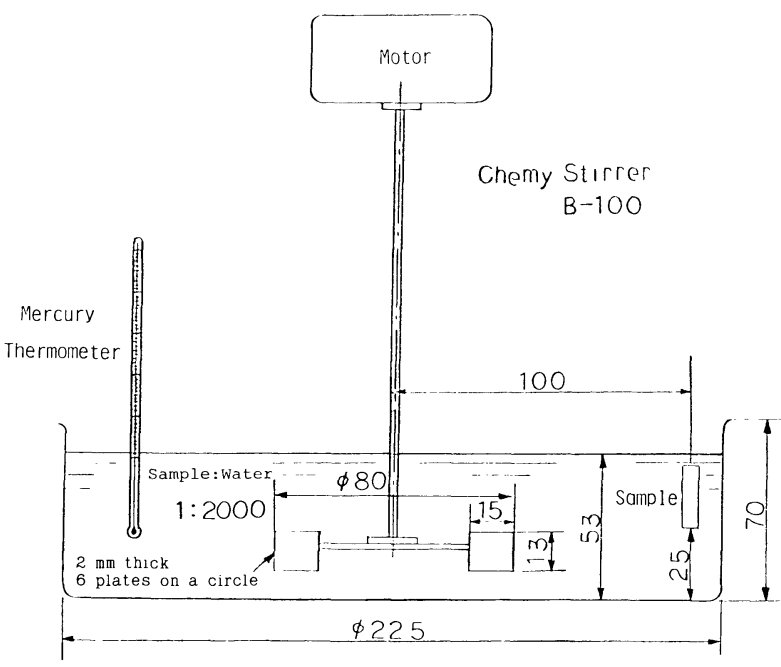

Fig. 1 Experimental apparatus for washing (unit in $\mathrm{mm}$ ) lute dry weight of the fabric. The amount of PVA on the fabric was $3.5 \%$ of the fabric weight. Fig. 1 shows the schmatic diagram of the apparatus used for the basic washing experments. The mercury thermometer $\left(0-100^{\circ} \mathrm{C}\right.$, a graduation; $0.1^{\circ} \mathrm{C}$ ) was removed during washing. The agitator rotated at $1,040 \mathrm{rpm}$ in all cases.

The sample washed beforehand at a given temperature for a certain time was immediately squeezed by a mangle with a squeezing ratio of $91 \%$. The residual PVA was analyzed using Hayashi's method ${ }^{[14]}$ as follows: the residual PVA was extracted 6 times in $30 \mathrm{cc}$ hot distilled water, and the extracts were diluted to lower than $100 \mathrm{ppm} .10 \mathrm{cc}$ of distilled water, $5 \mathrm{cc}$ of $38 \mathrm{~g} / \ell$ boric acid solution, and $1 \mathrm{cc}$ of $0.1 \mathrm{~N}$ iodine solution were added to $5 \mathrm{cc}$ of this solution in this order, and the solution was finally made to $25 \mathrm{cc}$ by adding distilled water. The absorbance of this solution was measured at $660 \mathrm{~nm}$, and the PVA content was quantitatively determined. This method was also applied to the samples which were undergone soaking in PVA solution and squeezed by a mangle, dried and not washed. From the PVA quantities obtained before and after washing, the residual ratio (PVA quantity before washing/PVA quantity after washing) were calculated. The soaking time was from 3 to 30 seconds. The temperature of the washing bath was 20,60 , and $90^{\circ} \mathrm{C}$.

\subsection{Repeated Washing Experiments}

After washing as discussed above, some samples were squeezed and put in the same bath again and the washing cycle was repeated. Others were left to stand for 60 seconds in air at the ambient temperature and washed again. Washing was repeated five times at the maximum with the soaking time for 5 seconds at $80^{\circ} \mathrm{C}$ and with the mangle squeezing ratio (Water weight on fabric after squeezing/fabric Weight) of $83 \%$ for each repeat.

\subsection{Washing by PVA Solution}

Washing experiments similar to procedure 2.2 were carried out with the soltions containing PVA 217, and the residual ratios of PVA were determined. The experimesntal washing conditions were: soaking time $=5,10,20$ seconds, temperature $=80^{\circ} \mathrm{C}$, mangle squeezing ratio $=83 \%$ and the PVA concentration in the washing bath $=0,0.5,1.0,1.5$, and $2.0 \%$.

\subsection{Washing by a Practical Washing Machine}

Fig. 2 shows a four-bath washing machine and Table 2 the specifications and the operational conditions used. The PVA concentration of the washing solution in each bath was measured every 5-30 minutes by Hayashi's method. As some samples were unfinished fabrics, we added $20 \mathrm{cc}$ of $2 \mathrm{~N}$ $\mathrm{HCl}$ and $50 \mathrm{cc}$ of distilled water to $5 \mathrm{cc}$ of the washing liquor to decompose the starch eluted therein, and boiled it for 90 minutes prior to PVA measurement. To analyse the test results with the practical washing machine, both unfinished and finished fabrics (desized, scoured, bleached and PVA 


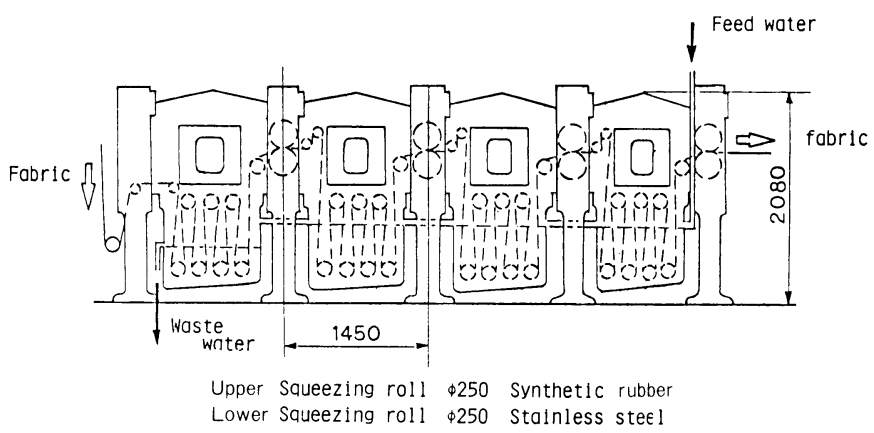

Fig. 24 Bath washing machine (unit in $\mathrm{mm}$ )

Table 2 Specifications and operational conditions of the washing machine

\begin{tabular}{llc}
\hline Operational speed & $(\mathrm{m} / \mathrm{min})$ & 90 \\
Water feed rate & $(\mathrm{t} / \mathrm{hr})$ & 2.4 \\
Volume of No. 1 washing bath & $(l)$ & 500 \\
Volume of No. 2 No. 4 washing bath & $(l)$ & 950 \\
Squeezing ratio of mangle & $(\%)$ & 85 \\
Washing temperature & $(\mathrm{C})$ & 80 \\
Dipping time in each bath & $(\mathrm{sec})$ & 5 \\
\hline
\end{tabular}

added) were tested by the same procedure as shown in Section 2.2 at $80^{\circ} \mathrm{C}$.

\section{Theory and Experimental Results}

\subsection{Basic Washing Experiments}

For the cylindrical body composed of fibers and water, the residual ratio of chemical is given by

$$
\frac{T(\mathrm{t})}{T_{0}}=\sum_{i=1}^{\infty} \sum_{j=1}^{\infty} A_{i j} \exp \left(-D_{f} \frac{\mu_{i j}{ }^{2}}{R^{2}} t\right)
$$

where: $A_{i j}=\left\{\frac{2 Q_{i}}{(1+\alpha) \beta^{2} \mu_{i j}^{2}}\right\}^{2} \frac{1}{1+\frac{\alpha}{1+\alpha} \cdot \frac{J_{1^{2}}\left(\mu_{i j}\right)}{J_{0}^{2}\left(\mu_{i j}\right)}}$

$J_{0}, J_{i}$; Bessel functions of zero and 1 st order

$Q_{i} ;$ root of $J_{0}\left(O_{i}\right)=0$

$$
\begin{aligned}
& \mu_{i j} ; \text { root of } Q_{i}=\beta \mu_{i j} \sqrt{1+\alpha \frac{2 J_{1}\left(\mu_{i j}\right)}{\mu_{i j} J_{0}\left(\mu_{i j}\right)}} \\
& \alpha ; \frac{\varepsilon}{(1-\varepsilon)} \frac{\nu_{f}}{\nu_{w}} \\
& \beta^{2} ; \frac{\lambda_{f}}{\lambda_{e}} \frac{\nu_{w}(1-\varepsilon) \rho^{2}}{\nu_{f} R^{2}} .
\end{aligned}
$$

$t$; washing time $(\mathrm{sec})$

$T_{0}, T(t)$; chemical quantity in a unit length of a fiber assembly at $t=0$ and $t(\mathrm{~g} / \mathrm{cm})$

$D_{f}$; Diffusion coefficient of the chemical in the fiber phase $\left(\mathrm{cm}^{2} / \mathrm{sec}\right.$.)

$\hat{\xi}$; Volume ratio of fiber $t_{0}$ a fiber assembly

(', $R$; radius of a fiber assembly and a fiber $(\mathrm{cm})$ $\nu_{f}, \nu_{w}$; specific concentration capacities of the fiber phase and of the water phase $\left(\mathrm{g} / \mathrm{cm}^{3 .}{ }^{\circ} \mathrm{E}\right)$

$i_{f}$; material transfer rate of a chemical in the fiber Phase $\left(\mathrm{g} / \mathrm{cm} \cdot \mathrm{sec}^{\circ} \mathrm{E}\right)$

$i_{e}$; effective material transfer rate in a fiber assembly $\left(\mathrm{g} / \mathrm{cm} \cdot \mathrm{sec} \cdot{ }^{\circ} \mathrm{E}\right)$

${ }^{\circ} E$ above is a potential unit and corresponds to the temperature in heat transfer. Therefore, $\nu_{f}$ and $\nu_{w}$ correspond to the volumetric specific heat, and $\lambda_{f}, \lambda_{e}$ to the heat conductivity. In the actual calculation, these were used as ratios and ${ }^{\circ} E$ did not appear.

Assuming that a fiber assembly is a plane board, a formula similar to eq. (1) can be derived ${ }^{[1]}$. However a washing test by stirred water proved to agree well to the case when the fiber assembly was assumed to be a column having the same radius as the yarn. So the case of a plane board was neglected.

As polymers such as PVA can not easily penetrate polyester fibers as well as cotton fibers, it is reasonable to put $\nu_{f}$ and $\alpha$ as zero, and so $\mu_{i j}$ turns into a one-dimensional series from a two dimensional infinite series. Therefore, $Q_{1}=\beta \mu_{i}$ and we have

$$
\frac{T(t)}{T_{0}}=\sum_{i=1}^{\infty} \frac{4}{Q_{i}{ }^{2}} \exp \left(-\frac{\lambda_{\theta}}{\nu_{w}} \frac{Q_{i}{ }^{2}}{(1-\varepsilon)} \bar{\rho}^{2} t\right) \cdots \cdots(2)
$$

This is similar to the equation derived under the assumption that the fiber assembly is a homogeneous column. So, as in the previous report, the second and the higher terms are extremely small except in the early stage of washing. Thus, we can change Eq. (2) to

$$
\begin{gathered}
\frac{T(t)}{T_{0}} \fallingdotseq \frac{4}{Q_{1}{ }^{2}} \exp \left(-\lambda_{e} \frac{Q_{1}{ }^{2}}{\nu_{w}} t\right) \cdots \cdots \cdots(3) \\
\log \frac{T(t)}{T_{0}^{-}} \fallingdotseq \log \frac{4}{Q_{1}{ }^{2}}-\log e \cdot \frac{\lambda_{e}}{\nu_{w}} \frac{Q_{1}{ }^{2}}{(1-\varepsilon) \rho^{2}} t \cdots(4)
\end{gathered}
$$

Eq. (4) shows linear relationship between the washing time $t$ and the $\log$ of the residual ratio of the chemical. Moreover,

$$
B=\log e \cdot \frac{\lambda_{e}}{\nu_{w}} \frac{Q_{1}{ }^{2}}{(1-\varepsilon) \rho^{2}}
$$

B is the slope of the graph and is a parameter for washing velocity.

Representing $D_{w}\left(\mathrm{~cm}^{2} / \mathrm{sec}\right)$ as the diffusion coefficient of a chemical in the water phase, and $\lambda_{w}\left(\mathrm{~g} / \mathrm{cm} \cdot \mathrm{sec} \cdot{ }^{\circ} \mathrm{E}\right)$ as the material transfer rate of a chemical in the water phase, we have

$$
D_{w}=\frac{\lambda_{w}}{\nu_{w}}
$$

So, $\lambda_{e} / \lambda_{w}$ in Eq. (3) becomes

$$
\frac{\lambda_{e}}{\nu_{w}}=\frac{\lambda_{e}}{\lambda_{w}} \frac{\lambda_{w}}{\nu_{w}}=\frac{\lambda_{e}}{\lambda_{w}} D_{w}
$$


From the volume ratio $s$ of a fiber in a fiber assembly, $\lambda_{e} / \lambda_{w}$ could be determined by the author's method ${ }^{[2]}$. This $s$ was calculated to be 0.65 from the observed thread diameter $\left(7.1 \times 10^{-3} \mathrm{~cm}\right)$, densities of polyester ${ }^{[18,19]}(1.38)$ and cotton (1.54), the swelling degree of cotton in water ${ }^{[20]}(0.43)$. These data led $\lambda_{e} / \lambda_{w}$, to be 0.21 .

The diffusion coefficient $D_{w}$ of PVA in water phase is $2.26 \times 10^{-7} \mathrm{~cm}^{2} / \mathrm{sec}^{[21]}$ (molecular weight $=8 \times 10^{4}$ at $20^{\circ} \mathrm{C}$ ). The diffusion coefficients at different temperatures were determined by Sato's method ${ }^{[22]}$. Table 3 shows these results.

Table 3 Diffusion coefficient of PVA in water phase and its effective diffusion coefficient in yarns

\begin{tabular}{cll}
\hline $\begin{array}{c}\text { Temperature } \\
\left({ }^{\circ} \mathrm{C}\right)\end{array}$ & $\begin{array}{l}\text { Diffusion coefficient } \\
\text { in water phase } \\
D_{w}=\lambda_{w} / \nu_{w} \\
\left(\mathrm{~cm}^{2} / \mathrm{sec}\right)\end{array}$ & $\begin{array}{l}\text { Effective diffusion } \\
\text { coefficient in yarns } \\
\lambda_{e} / \nu_{w} \\
\left(\mathrm{~cm}^{2} / \mathrm{sec}\right)\end{array}$ \\
\hline 20 & $2.6 \times 10^{-7}$ & $0.55 \times 10^{-7}$ \\
60 & $4.6 \times 10^{7}$ & $0.97 \times 10^{7}$ \\
90 & $6.1 \times 10^{-7}$ & $1.28 \times 10^{7}$ \\
\hline
\end{tabular}

Figure 3 shows the basic washing experiment. At all temperatures, a linear relationship between $\log \left(T / T_{0}\right)$ and $t$ is evident. Table 4 shows the $B$ values obtained from Fig. 3 and derived from Eq. (5), indicating that our theory based on the diffusion theory is reasonable, that the assumed effective diffusion coefficient is sound, that PVA can not penetrate the fiber, that water behaves violently outside the yarn when stirred. However, the experimental values of $B$ are a little bit larger than the theoretical values, showing that the washing velocity is higher than the calculated. In other words, the agitation proves to be effective to make the apparent yarn diameter required for the chemical diffusion to be smaller than the observed diameter.

The intersection of the $t-\log \left(T / T_{0}\right)$ line with the $y$-axis is lower than the value derived from Eq. (4) $\left(\log 4 / Q_{1}{ }^{2}=\right.$

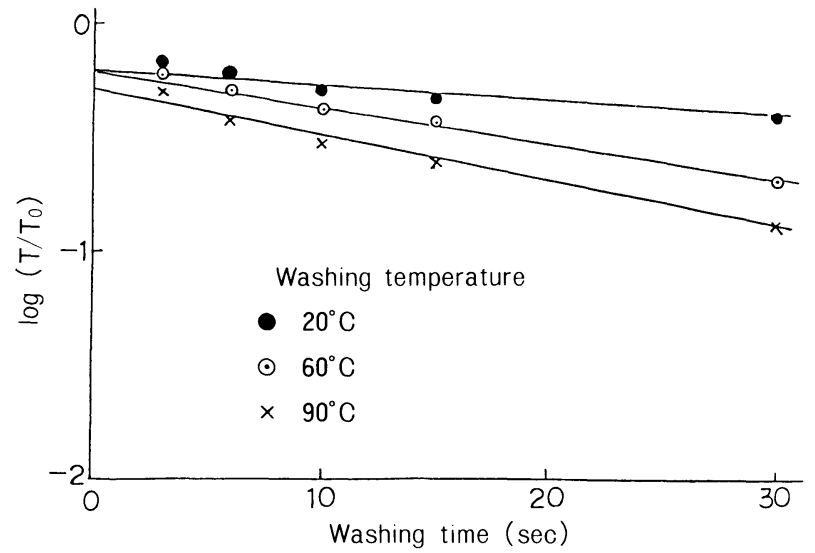

Fig. 3 Relation between washing time and residual ratio of PVA
Table $4 \mathrm{~B}$ values observed and calculated

\begin{tabular}{lll}
\hline $\begin{array}{l}\text { Temperrature } \\
\left({ }^{\circ} \mathrm{C}\right)\end{array}$ & $\begin{array}{c}\text { Observed B } \\
\left(\mathrm{sec}^{1}\right)\end{array}$ & $\begin{array}{c}\text { Calculated B } \\
\left(\mathrm{sec}^{-1}\right)\end{array}$ \\
\hline 20 & 0.008 & 0.008 \\
60 & 0.016 & 0.014 \\
90 & 0.020 & 0.018 \\
\hline
\end{tabular}

$-0.16)$. This is attributed to the fact that the residual ratio of chemical decreases as a whole after squeezing. The experiment using caustic soda shows that this residual ratio decreased by $2 / 3$ after squeezing ${ }^{[3]}$. If so, the intersection lis at $\log \left(4 / Q_{2} \cdot 2 / 3\right)=-0.34$. The difference between this and the experiment could be explained by the fact that dry PVA adhered to the fiber before washing, makes the washing time longer by dissolvation as compared when wet. This will be further elaborated in the next section.

\subsection{Repeated Washing Experiment}

Figure 4. shows the relationship between the number of washing and the logarithm of residual ratio of PVA after washing. The represents the results when fabrics were immediately put into the washing bath after soaking-squeezing. The $\times$ represents the results when fabrics were first kept in air for 60 seconds at the ambient temperature.

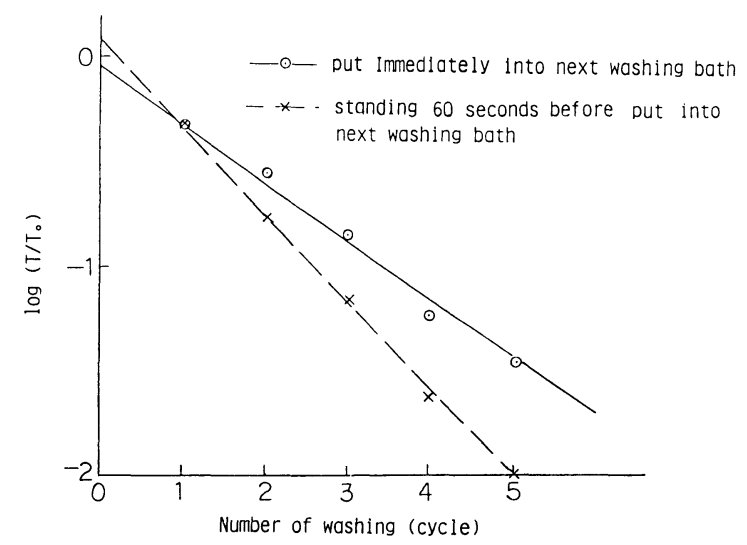

Fig. 4 Relation between number of washing and residual PVA. Washing temperature: $80^{\circ} \mathrm{C}$ Washing times: $5 \mathrm{sec} /$ cycle

In both cases, the linear relationship is obtained. This is the same as obtained in the removal of caustic soda from cotton fabrics. This is formulated as

$$
y=A E^{n}
$$

where $y$ is the residual ratio of chemical in the fabric to that before washing, $n$ is the number of washing, $A$ and $E$ are constants. The $\boldsymbol{A}$ value is due to the difference in the distribution of the chemical concentration among each washing. $A E$ represents the residual ratio of the chemical in the first washing stage, while $E$ is that in the after washing stage. 
In the case of washing immediately after soaking and squeezing, the distribution of a chemical concentration in the radial direction of a yarn is uniform before the first washing. But the chemical on the yarn surface is removed rapidly in the first washing stage. Therefore, the concentration of a chemical on the fiber surface is already small before the second washing, and the rapid removal does not take place later washing.

If a yarn is assumed as a homogeneous column, the concentration distribution along the radii is expressed by the 0th order Bessel function except in the early washing stage. The rapia decrease of the 2 nd term and the higher terms in Eq. (2) with increasing time means that the distribution of a chemical concentration changes from a uniform ${ }^{[3]}$ distribution to the 0th order Bessel function. The same behaviour is observed in PVA washing. If the distribution pattern of concentration does not change after squeezing, $A$ becomes $4 / Q_{1}{ }^{2}=0.69$. The intercept of y axis in Fig. 3 does not give this $A$ value, but gives -0.04 , which is much closer to zero than $\log 0.69=-0.16$. The previous paper on washing of caustic soda from contton fabrics ${ }^{[3]}$ showed a very near value to -0.16 . This is because that in washing of caustic soda the fiber assembly was treated without drying after squeezing. On the other hand, in case of PVA, dry PVA attached on the fiber assembly consumed much time to be disolved into water, and made the first washing not so effective and led $A$ near 1 .

When the sample is left as it is for $60 \mathrm{sec}$, the effect of each washing increases and the residual ratio of chemical decreases. This may be because the distribution of PVA concentration in the yarn changes from the Bessel function type to a uniform distribution because of PVA moving to the yarn surface from the inside of yarn in $60 \mathrm{sec}$ standing and being removed rapidly as well as first washing stage. The reason that the intercept with $\mathrm{y}$ axis becomes plus is because that first washing is uneffective due to much longer Time of dry PVA disolving.

When a fiber assembly was washed immediately after squeezing, the residual ratio of chemical is $52 \%$. The experimental $B$ value, shown in Table 4 and the washing effect of spueezing made the residual ratio of PVA calculable as $56 \%$ at $60^{\circ} \mathrm{C}$ and $53 \%$ at $90^{\circ} \mathrm{C}$. These values show relatively good agreement.

\subsection{Washing experiment by PVA solution}

Barish $^{[15]}$, Bonkalo ${ }^{[16]}$, Ratna Prabhu ${ }^{[17]}$ and we derived theoretical equations on the contamination of washing water by chemicals flowed out from the fiber assembly. However, as some coditions were too simlified, we will treat the ploblem more theoretically.

The following symbols are defined, the suffices to which are $o, a, b, c$, but expressed by $m$, and mean before washing, immediately after dipping, after washing (before squeezing)

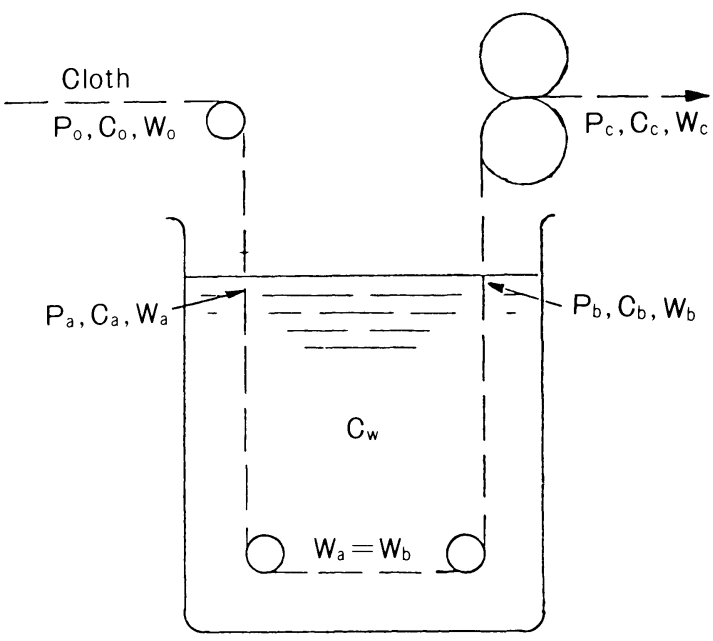

Fig. 5 Notations used

and after squeezing, respectively:

$P_{0}, P_{a}, P_{b}, P_{c}$ : quantity of a chemical in the fiber assembly per unit weight of the fiber assembly.

$W_{m}$ : Water content in the fiber assembly $\left(\mathrm{cm}^{3} / \mathrm{g}\right)$, and $W_{a}=$ $W_{b}$

$C_{m}$ : Concentration of a chemical in the water phase contained in the fiber assembly $\left(\mathrm{g} / \mathrm{cm}^{3}\right)$.

$q_{m}$ : Weight of a chemical absorbed in the unit fiber assembly

$C_{w}$ : Concentration of a chemical in the washing liquid $\left(\mathrm{g} / \mathrm{cm}^{3}\right)$

$E_{0}, E\left(C_{w}\right)$ : Residual ratio of chemical on the fiber assembly $\left(\boldsymbol{P}_{c} / \boldsymbol{P}_{0}\right)$ after washing by pure water and washing by liquid (the concentration of a chemical is $C_{w}$ )

Following assumptions are put for analysis.

(1) The quantity of a chemical in a fiber is related to the concentration in the liquid around the fiber, and

$$
q_{m}=C_{m} K(K: \text { constant })
$$

(2) Releasing velocity of a chemical from the fiber assembly into water is related to the concentration difference of a chemical between inside and outside of the fiber assembly. Thus, if washing was done at the same condition except $C_{a}$, $C_{b}$, and $C_{u}$, we have

$$
\frac{C_{b}-C_{w}}{C_{a}-C_{w}}=K(K: \text { constant })
$$

(3) When water in the fiber assembly was squeezed out by a mangle, the chemical amount in the fiber assembly (except the chemical absorbed by fibers) decreases proportionally to the water content of the fiber assembly.

Generally,

$$
\begin{aligned}
P_{m} & =q_{m}+C_{m} W_{m} \\
& =C_{m}\left(k+W_{m}\right)
\end{aligned}
$$


So, putting this equation into Eq. (9), we have

$$
\frac{\frac{P_{b}}{k+W_{a}}-C_{w}}{\frac{P_{a}}{k+W_{a}}-C_{w}}=K
$$

Also, from the assumption (3),

$$
\begin{aligned}
& P_{a}=P_{0}+\left(W_{a}-W_{0}\right) C_{w} \\
& P_{b}=P_{c}+\left(W_{a}-W_{c}\right) C_{c}
\end{aligned}
$$

Combining with Eq. (10) and Eq. (11), we have

$$
\frac{\frac{P_{c}}{k+W_{c}}-C_{w}}{\frac{P_{0}+\left(W_{a}-W_{0}\right) C_{w}}{k+W_{a}}-C_{w}}=K
$$

Eq. (12) and $E_{0}, E\left(C_{w}\right)$ lead to

$$
E=E_{0}+\left\{k+W_{c}-E_{0}\left(W_{0}+k\right)\right\} \frac{C_{w}}{P_{0}}
$$

If $W_{0}=W_{c}$, we have from Eqs. (10) and (13),

$$
E=E_{0}\left(\frac{C_{0}-C_{w}}{C_{0}}\right)+\frac{C_{w}}{C_{0}}
$$

This result agrees with that of washing of caustic soda ${ }^{[4}$. When the sample fiber assembly was dried before washing and PVA had no preferential adsorption to the fiber assembly, $W_{0}$ anf $k$ are represented by

$$
W_{0}=0, \quad k=0
$$

So, Eq. (15) is obtained.

$$
E=E_{0}+\frac{C_{w} W_{c}}{P_{0}}
$$

Fig. 6 shows the relationship between the residual ratio of PVA and PVA concentration in the washing liquid.

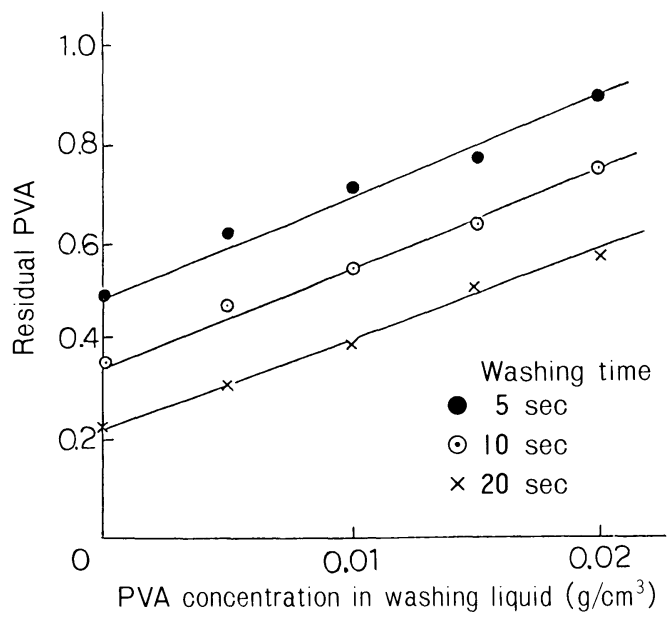

Fig. 6 Relation between residual ratio of PVA and PVA concentration in washing liquid. (Washing temperature: $80^{\circ} \mathrm{C}$ )
It proves Eq. (15) because the three kinds of experimental results were expressed by straight lines with the same slope (ca. 18-20) and values are very close to the calculated values, $W_{c} / P_{0}=0.83 / 0.035 \fallingdotseq 24$.

\subsection{Washing Experiment by a Practical Machine}

Hitherto, we had good agreement between the theory and experimental results. Using this theory, we want to check the washing experiment done by a multi-both washing machine.

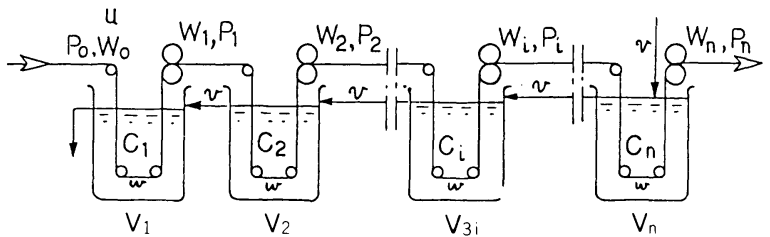

Fig. 7 Notations used

The following notations were used as shown in Fig. 7.

$t_{u}$; time after a washing machine is started (sec.)

$\boldsymbol{P}_{\mathbf{0}}, \boldsymbol{P}_{i}\left(t_{u}\right)$; Quantity of a chemical in the fabric before washing and after the $i$ th bath after $t_{w}$ )

$C_{i}\left(t_{x}\right)$ : Concentration of a chemical in the washing liquid in the $i$ th bath $\left(\mathrm{g} / \mathrm{cm}^{3}\right)$

$v$; volume of washing liquid $\left(\mathrm{cm}^{3} / \mathrm{sec}\right)$

$V_{i}$; Volume of the $i$ th bath $\left(\mathrm{cm}^{3}\right)$

$F$; Weight of fabric per unit length $(\mathrm{g} / \mathrm{cm})$

$u$ : Transfer velocity of fabric $(\mathrm{cm} / \mathrm{sec})$

$k_{i}$; Residual ratio of a chemical in the $i$ th bath when a fabric was washed (before squeezing) by washing liquid (concentration of chemical is 0 .)

$W_{0}, w$; Water content of fabrics before washing and in each bath $\left(\mathrm{cm}^{3} / \mathrm{g}\right)$

$W_{i}$; Water content of fabrics after squeezed with the mangle in the $i$ th bath.

Fresh water was fed only into the last bath, and was flown up into the preceeding bath continuously, and was finally exhausted from No. 1 bath. The amount of a chemical adsorbed on to a fiber was assumed as related proportionally to the concentration of a chemical in the water phase around the fiber.

Put eq. (12) to No. 1 bath and we have

$$
\begin{aligned}
P_{1} & =\frac{K_{1}\left(W_{1}+k\right)}{w+k} P_{0}+\left(W_{1}+k\right) \\
& \times\left\{1-\frac{K_{1}\left(W_{\mathbf{0}}+k\right)}{w+k}\right\} C_{1} \quad \ldots .
\end{aligned}
$$

Similarly, from Eq. (12) and Eq. (16),

$$
\begin{aligned}
P_{2} & =\frac{K_{1} K_{2}\left(W_{1}+k\right)\left(W_{2}+k\right)}{(w+k)^{2}} P_{0} \\
& +\frac{K_{2}\left(W_{1}+k\right)\left(W_{2}+k\right)}{w+k}\left\{1-\frac{K_{1}\left(W_{0}+k\right)}{w+k}\right\} C_{1} \\
& +\left(W_{2}^{r}+k\right)\left\{1-\frac{K_{2}\left(W_{1}+k\right)}{w+k}\right\} C_{2} \cdots \cdots \cdots \cdots(17
\end{aligned}
$$




$$
\begin{aligned}
& P_{3}=\frac{K_{1} K_{2} K_{3}\left(W_{1}+k\right)\left(W_{2}+k\right)\left(W_{3}+k\right)}{(w+k)^{3}} P_{0} \\
& +\frac{K_{2} K_{3}\left(W_{1}+k\right)\left(W_{2}+k\right)\left(W_{3}+k\right)}{(w+k)^{2}} \\
& \times\left\{1-\frac{K_{1}\left(W_{0}+k\right)}{w+k}\right\} C_{1} \\
& \frac{K_{3}\left(W_{2}+k\right)\left(W_{3}+k\right)}{w+k}\left\{1-\frac{K_{2}\left(W_{1}+k\right)}{w+k}\right\} C_{2} \\
& +\left(w_{3}+k\right)\left\{1-\frac{K_{3}\left(W_{2}+k\right)}{w+k}\right\} C_{3} \\
& P_{n}=\frac{K_{1} K_{2} \cdots K_{n}\left(W_{1}+k\right)\left(W_{2}+k\right) \cdots\left(W_{n}+k\right)}{(w+k)^{n}} P_{0} \\
& +K_{2} K_{3} \cdots K_{n}\left(W_{1}+k\right)\left(W_{2}+k\right) \cdots\left(W_{n}+k\right) \\
& \times\left\{1-\frac{K_{1}\left(W_{0}+k\right)}{w+k}\right\} C_{1} \\
& \frac{K_{3} K_{4} \cdots K_{n}\left(W_{2}+k\right)\left(W_{3}+k\right) \cdots\left(W_{n}+k\right)}{(w+k)^{n-2}} \\
& \left\{1-\frac{K_{2}\left(W_{1}+k\right)}{w+k}\right\} C_{2} \\
& \left(W_{n}+k\right)\left\{1-\frac{K_{n}\left(W_{n-1}+k\right)}{w+k}\right\} C_{n}
\end{aligned}
$$

By considering the mass balance of a chemical in the 1st bath and from Eq. (16),

$$
\begin{aligned}
V_{1} \frac{d C_{1}}{d t_{w}} & =F u P_{0}-F u P_{1}-\left\{v-F u\left(W_{n}-W_{0}\right)\right\} C_{1} \\
& +\left\{v-F u\left(W_{n}-W_{1}\right)\right\} C_{2} \\
V_{1} \frac{d C_{1}}{d t_{w}} & =F u\left\{1-\frac{K_{1}\left(W_{1}+k\right)}{w+k}\right\} P_{0} \\
& -F u\left(W_{1}+k\right)\left\{1-\frac{K_{1}\left(W_{0}+k\right)}{w+k}\right\} C_{1} \\
& -\left\{v-F u\left(W_{n}-W_{0}\right)\right\} C_{1} \\
& +\left\{v-F u\left(W_{n}-W_{1}\right)\right\} C_{2} \quad \cdots \ldots \ldots \ldots \ldots(20)
\end{aligned}
$$

By same way, Eq. (21)-Eq. (24) are obtained.

$$
\begin{aligned}
V_{2} \frac{d C_{2}}{d t_{w}}= & F u \frac{K_{1}\left(W_{1}+k\right)}{w+k}\left\{1-\frac{K_{2}\left(W_{2}+k\right)}{w+k}\right\} P_{0} \\
+ & F u\left(W_{1}+k\right)\left\{1-\frac{K_{2}\left(W_{2}+k\right)}{w+k}\right\} \\
& \times\left\{1-\frac{K_{1}\left(W_{0}+k\right)}{w+k}\right\} C_{1} \\
& -F u\left(W_{2}+k\right)\left\{1-\frac{K_{2}\left(W_{1}+k\right)}{w+k}\right\} C_{2} \\
& -\left\{v-F u\left(W_{n}-W_{1}\right)\right\} C_{2} \\
+ & \left\{v-F u\left(W_{n}-W_{2}\right)\right\} C_{3} \quad \cdots \cdots \cdots \cdots \cdots(21) \\
V_{3} \frac{d C_{3}}{d t_{w}}= & F u \frac{K_{1} K_{2}\left(W_{1}+k\right)\left(W_{2}+k\right)}{(w+k)^{2}} \\
& \times\left\{1-\frac{K_{3}\left(W_{3}+k\right)}{w+k}\right\} P_{0} \\
+ & F u \frac{K_{2}\left(W_{1}+k\right)\left(W_{2}+k\right)}{w+k} \\
& \times\left\{1-\frac{K_{3}\left(W_{3}+k\right)}{w+k}\right\}\left\{1-\frac{K_{1}\left(W_{0}+k\right)}{w+k}\right\} C_{1}
\end{aligned}
$$

$$
\begin{aligned}
& +F u\left(W_{2}+k\right)\left\{1-\frac{K_{3}\left(W_{3}+k\right)}{w+k}\right\} \\
& \times\left\{1-\frac{K_{2}\left(W_{1}+k\right)}{w+k}\right\} C_{2} \\
& -F u\left(W_{3}+k\right)\left\{1-\frac{K_{3}\left(W_{2}+k\right)}{w+k}\right\} C_{3} \\
& -\left\{v-F u\left(W_{n}-W_{2}\right)\right\} C_{3} \\
& +\left\{v-F u\left(W_{n}-W_{3}\right)\right\} C_{4} \\
& V_{i} \frac{d C_{i}}{d t_{w}}=F u \frac{K_{1} K_{2} \cdots K_{i-1}\left(W_{1}+k, W_{2}+k\right) \cdots\left(W_{i-1}+k\right)}{(w+k)^{i-1}} \\
& \times\left\{1-\frac{K_{i}\left(W_{i}+k\right)}{w+k}\right\} P_{0} \\
& +F u \frac{K_{2} K_{3} \cdots K_{i-1}\left(W_{1}+k\right)\left(W_{2}+k\right) \cdots\left(W_{i-1}+k\right)}{\left(\overline{w+k)^{i-2}}\right.} \\
& \times\left\{1-\frac{K_{i}\left(W_{i}+k\right)}{w+k}\right\}\left\{1-\frac{K_{1}\left(W_{0}+k\right)}{w+k}\right\} C_{1} \\
& +F u \frac{K_{3} K_{4} \cdots K_{i-1}\left(W_{2}+k\right)\left(W_{3}+k\right) \cdots\left(W_{i-1}+k\right)}{(w-k)^{i-3}} \\
& \times\left\{1 \ldots \frac{K_{i}\left(W_{i}+k\right)}{w+k}\right\}\left\{1-\frac{K_{2}\left(W_{1}+k\right)}{w+k}\right\} C_{2} \\
& -F u\left(W_{i-1-i} k\right)\left\{1-\begin{array}{c}
K_{i}\left(W_{i}+k\right) \\
w+k
\end{array}\right\} \\
& \times\left\{1-\frac{K_{i-1}\left(W_{i-2}+k\right)}{w+k}\right\} C_{i-1} \\
& -F u\left(W_{i}+k\right)\left\{1-\frac{K_{i}\left(W_{i-1}+k\right)}{w+k}\right\} C_{i} \\
& -\left\{v-F u\left(W_{n}-W_{i-1}\right)\right\} C_{i} \\
& +\left\{v-F u\left(W_{n}-W_{i}\right)\right\} C_{i+1}
\end{aligned}
$$

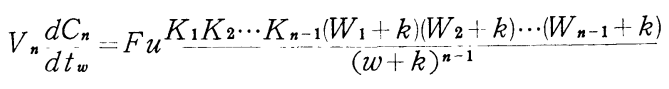

$$
\begin{aligned}
& \times\left\{1-\frac{K_{n}\left(W_{n}+k\right)}{w+k}\right\} P_{0} \\
& +F u \frac{K_{2} K_{3} \cdots K_{n-1}\left(W_{1}+k\right)\left(W_{2}+k\right) \cdots\left(W_{n-1}+k\right)}{(w+k)^{n-2}} \\
& \times\left\{1-\frac{K_{n}\left(W_{n}+k\right)}{w+k}\right\}\left\{1-\frac{k_{1}\left(W_{0}+k\right)}{w+k}\right\} C_{1} \\
& +F u \frac{K_{3} K_{4} \cdots K_{n-1}\left(W_{2}+k\right)\left(W_{3}+k\right) \cdots\left(W_{n-1}+k\right)}{(w+k)^{n-3}} \\
& \times\left\{1-\frac{K_{n}\left(W_{n}+k\right)}{w+k}\right\}\left\{1-\frac{K_{2}\left(W_{1}+k\right)}{w+k}\right\} C_{2} \\
& +F u\left(W_{n-1}+k\right)\left\{1-\frac{K_{n}\left(W_{n}+k\right)}{w+k}\right\} \\
& \times\left\{1-\frac{K_{n-1}\left(W_{n-2}+k\right)}{w+k}\right\} C_{n-1} \\
& -F u\left(W_{n}+k\right)\left\{1-\frac{K_{n}\left(W_{n-1}+k\right)}{w+k}\right\} C_{n} \\
& -\left\{v-F u\left(W_{n}-W_{n-1}\right)\right\} C_{n}
\end{aligned}
$$

By solving these Eqs., $C_{1}, C_{2}, \ldots C_{n}$ are obtained. Then, putting these values into Eqs.(16), (17), (18), (19), $P_{1}, P_{2}, \ldots$ $P_{i}, \ldots P_{n}$ are obtained. However, as it is very difficult to solve these equations universally, we solve them numerically by the Runge Kutta method and get $C_{i}$ and $P_{i}$. 


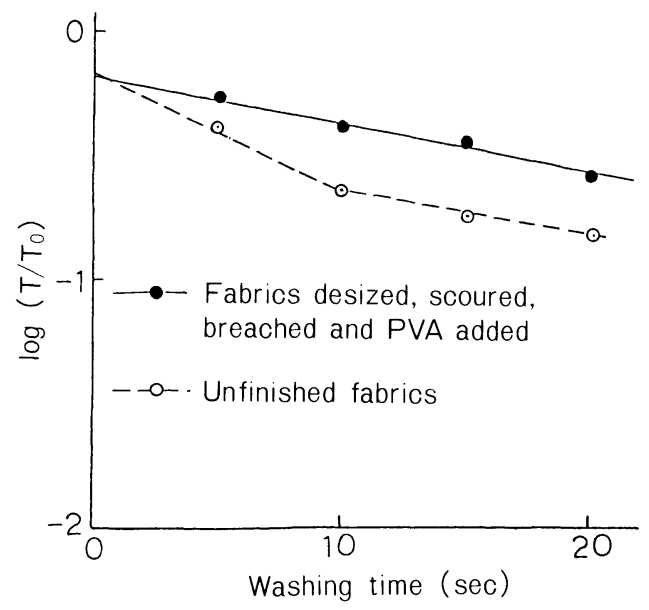

Fig. 8 Relation between residual ratio of PVA and washing time. (Washing temperature: $80^{\circ} \mathrm{C}$ )

We carried out the fundamental washing experiment by using unfinished fabrics, and the result were compared with those of the fabrics which were designed scoured, bleached and PVA added. Fig. 8 shows those results indicating better washing results. But washing out rate for unfinished fabric is larger than that of treated one until washing time becomes $10 \mathrm{sec}$, after which both untreated and treated fabrics have the same washing out rate. Parameter $B$ which expresses the washing rate is caluclated as $-0.0185 \mathrm{sec}^{-1}$ from the log $\left(T_{0} / T\right)$ vs washing time curve within the $10-20 \mathrm{sec}$ reagion. This value agrees with that theoretically calculated from the diffusion coefficient of PVA $\left(-0.0166 \mathrm{~cm}^{-1}\right)$ at $80^{\circ} \mathrm{C}$.

The rapid washing out of PVA until $10 \mathrm{sec}$ may be caused by that PVA attached on the yarn surface can disolve into water at a very early stage. Practically, unfinished fabrics have a large amount of PVA on the yarn surface. A chemical attached on the yarn surface is generally washed out very rapidly at beginning of washing. But in the case of PVA, rapid washing out continues for $10 \mathrm{sec}$. This is because yarns treated by PVA were dried and so it took more time to disolve the dried PVA on the fiber surface into water.

If the residual ratio of PVA becomes $2 / 3$ by squeezing, parameter $B$ of unfinished fabrics is calculated as 0.047 $\mathrm{sec}^{-1}$ from the washing out rate in the $0-10 \mathrm{sec}$. This value is used in the following discussion.

Fig. 9 compares the theoretical values with those experimented in which,

$$
\begin{aligned}
& K_{1}=K_{2}=10^{-B t}=10^{-0.047 \times 5} \fallingdotseq 0.58 \\
& n=4 \\
& K_{3}=K_{4}=10^{-B t}=10^{-0.017 \times 5}=0.82 \\
& k=0: \text { no prefarential adsorption } \\
& w_{0}=0: \text { dry fabrics were used } \\
& w=1.25: \text { observed value }
\end{aligned}
$$

The values of $\boldsymbol{P}_{0}, v, V_{n}, F, u$; were shown in Tables 1 and 2.

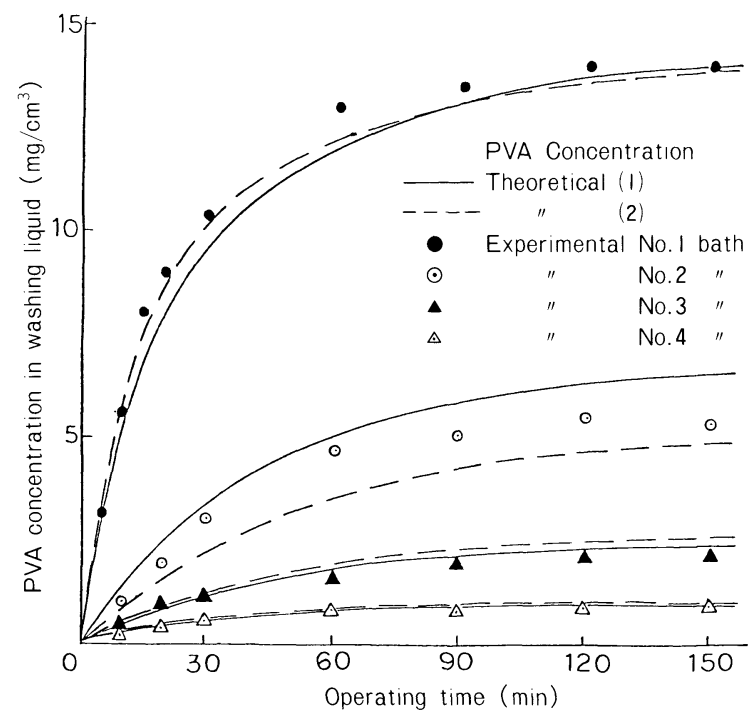

Fig. 9 Relation between operating time of washing machine and PVA concentration in washing liquid.

Fig. 9 shows the excellent agreement between theoretical and experimental values. The reason that the test results of No. 2 are all lower than those of the theory is explaind by the following facts. In Fig. 8, the washing out rates for No. 1 and No. 2 baths were large and the value $B$ in these baths was taken as $0.047 \mathrm{sec}$, but in a practical washing machine the washing out rate of No. 2 bath is not so large as that of No. 1 value. The difference between the basic experimental data (Fig. 8) and those by the practical washing machine may be because that the stirring condition in the practical washing bath has a large effect on washing out of PVA attached on the yarn surface. In Fig. 9, broken lines show theoretical values obtained by the assumption that PVA on the yarn surface which was washed out rapidly in Fig. 8 is completely washed out in No. 1 bath. In this case, the theoretical concentration in No. 2 bath becomes much smaller than that observed. Therefore, the practical washing may be carried out on the condition between these two extreme assumptions.

Table 5 shows the residual ratio of PVA on the fabrics, showing a good agreement. We dealt here with the continuous changing of the chemical concentration, and $C_{i}$ is obtained by setting $d C_{i} / d t_{w}=0$ in Eqs. (16)-(24) in the dynamic equilibrium state. Then, the residual ratio of chemical after washing at each bath is calculated by $C_{i}$ and Eqs. (16)(19).

Table 5 Residual ratio of PVA on the fabric

\begin{tabular}{lll}
\hline $\begin{array}{c}\text { Operating time } \\
(\mathrm{min})\end{array}$ & Observed value & Calculated value \\
\hline 30 & 0.090 & 0.087 \\
180 & 0.112 & 0.114 \\
\hline
\end{tabular}




\section{Conclusion}

(1) Washing of PVA from polyester/cotton blended fabrics was investigated by on theory and experiments. Theoretical results show good agreement with those of experimental results based on the following two assumptions.

Assumption 1: Under the condition used for the fundadamental experiment in this report, water in the bath and intra yarns is completely agitated, and fresh water contacts with yarns. The fiber assembly is assumed as a column, the diameter of which is the same as that of the yarn.

Assumption 2: PVA does not enter the fibers of polyester and cotton.

(2) When washing is repeated under the same condition, the washing out efficiency per washing becomes the same after the 2 nd washing process. The high washing out efficiency at the 1 st washing process is because of the uniform distribution of a chemical in the sample.

(3) The theoretical equations for washing in the solution contaminated by substances being dropped from textiles were discussed and derived. The results of washing by PVA solution and caustic soda ${ }^{[4]}$ agreed well with the theoretical values derived in this paper.

(4) The washing experiment by a practical continuous washing machine is discussed by the theoretical equations. We got a good agreement. The equations obtained on these views can be applied not only to the case of dynamic equilibrium but also to the case of continuous changing of chemical concentration. They give the best washing conditions with minimum energy and highest efficiency.

\section{References}

[1] H. Matsui, K. Moriyama and S. Fukuoka; J. Text. Mach. Soc. Japan, 30, 214 (1977).
[2] H. Matsui and K: Moriyama; J. Text. Mach. Soc. Japan, 31, 18 (1978).

[3] H. Matsui, M. Kobayashi and K. Koji; J. Text. Mach. Soc. Japan, 29, 108 (1976).

[4] H. Matsui; Textbook of Dyeing and Finishing Technology course, p. 41, 1977, Text. Mach. Soc. Japan.

[5] T. Wakita; Kiso Sen-i Kougaku, Vol. 5, p. 197, 1971. Text. Mach. Soc. Japan.

[6] Y. Konishi and T. Wakita; Ann. Rep. Gakushin 120 Comm., 10, p. 136 (1958).

[7] ibid., 12, p. 282 (1960).

[8] ibid., 13, p. 190 (1961).

[9] ibid., 14, p. 102 (1962).

[10] ibid., 15, p. 179 (1963).

[11] ibid., 16, p. 186 (1964).

[12] ibid., 17, p. 208 (1965).

[13] ibid., 18, p. 185 (1966).

[14] S. Hayashi; Polymer Applications, 18, 739 (1969).

[15] G. J. Parish; J. Soc. Dyers. Col., 78, 109 (1962).

[16] T. Bankálo; Textil-Praxis, 22, 510 (1967).

[17] M. Ratna Prabhu, P. B. Thala and K. Subrahmanyam; J. Soc. Dyers. Col., 86, 368 (1970).

[18] Zenkoku Sen-i Kogyo Kyoriyoku Kai; Orimoto Bunkaisetsukei no Jissai Chishiki, p. 168, 1960 Sen-i Gijitsu Kenkyusha, Japan.

[19] Text. Mach. Soc. Japan; Sen-i Sooran, p. 15, 1970.

[20] Soc. Fib. Sci. Tech. Japan; Sen-i Butsuri Gaku, p. 181, 1965, Maruzen, Japan.

[21] A. Nakajima and M. Hosono; Koobunshi Busei, Vol. 2, p. 345, 1969 Kagaku Doojin, Japan.

[22] K. Satoo; Busei Jyosu Suisan Hoo, p. 307, 1958 Maruzen, Japan. 